\title{
A Robust Method for MR Image Segmentation and Multiple Scleroses Detection
}

\author{
H. Zouaoui ${ }^{1, *}$, A. Moussaoui ${ }^{1}$, M. Oussalah ${ }^{2}$, and A. Taleb-Ahmed ${ }^{3}$ \\ ${ }^{1}$ Computer Science Department, Ferhat Abbas University, Algeria \\ ${ }^{2}$ Centre for Ubiquitous Computing, Faculty of Information Technology, University of Oulu, Finland \\ ${ }^{3}$ LAMIH Laboratory University of Valenciennes France
}

\begin{abstract}
In the present article, we propose a new approach for the segmentation of the MR images of the Multiple Sclerosis (MS). The Magnetic Resonance Imaging (MRI) allows the visualization of the brain and it is widely used in the diagnosis and the follow-up of the patients suffering from MS. Aiming to automate a long and tedious process for the clinician, we propose the automatic segmentation of the MS lesions. Our algorithm of segmentation is composed of three stages: segmentation of the brain into regions using the algorithm Fuzzy Particle Swarm Optimization (FPSO) in order to obtain the characterization of the different healthy tissues (White matter, grey matter and cerebrospinal fluid (CSF)) after the extraction of white matter (WM), the elimination of the atypical data (outliers) of the white matter by the algorithm Fuzzy C-Means (FCM), finally, the use of a Mamdani-type fuzzy model to extract the MS lesions among all the absurd data.
\end{abstract}

Keywords: Multiple Sclerosis, Magnetic Resonance Imaging, Segmentation, Fuzzy C-Means, Particle Swarm Optimization, Fuzzy Controller.

\section{INTRODUCTION}

Multiple sclerosis (MS) is a chronic inflammatory demyelinating disease of the central nervous system [1,2]. This disease is associated with brain tissue damage (e.g., lesions) that can be observed through Magnetic Resonance Imaging (MRI). These lesions can appear as a hyperintense or a hypointense signal depending type of MRI sequence employed and observed properties [3]. Magnetic resonance (MR) imaging has several advantages over other medical imaging modalities, including the high contrast among different soft tissues [4]. In this article, we are interested in the brain MRI analysis conducted in a community of patients suffering from Multipe Sclerosis (MS).

Diagnosis of magnetic resonance imaging (MRI) is usually based on scanning of MRI. However, a challenge arises due to the "normal-appearing white matter (WM)" problem, which causes the lesions within the WM to appear the same as healthy WMs. Even experienced neuroradiologist may not perceive the differences [6].

Strictly speaking, MRI has brought in several benefits to the study of Multiple Sclerosis (MS). It provides accurate measurement of disease activity, facilitates precise diagnosis, and aid in the assessment of newer therapies [5]. MRI also played a key role in the patient's follow-up state and the evaluation of the prescribed treatment. Therefore, automatic extraction of potential

*Author to whom correspondence should be addressed. quantifiers for the Multiple Sclerosis has many prospective applications in both clinical and pharmaceutical fields [7]. The appearance of new lesions or the raising of ancient patches detected by MRI constitute one of the acknowledged criteria for definitive diagnosis [8]. Besides, MR imaging is often used to characterize and quantify multiple sclerosis (MS) lesions in the brain and spinal cord. The number and volume of lesions have been used to evaluate MS disease burden, to track the progression of the disease and to evaluate the effect of new pharmaceuticals in clinical trials. Accurate identification of MS lesions in MR images is extremely difficult due to variability in lesion location, size and shape in addition to anatomical variability between subjects. Since manual segmentation requires expert knowledge; it is time consuming and it is subject to intra- and inter-expert variability, many methods have been proposed to automatically segment lesions [9].

The segmentation of various tissues and structures in medical images in a robust and efficient manner is of crucial significance in many applications, such as the identification of brain pathologies from Magnetic Resonance (MR) images [10]. Indeed, image segmentation is generally the most important stage in the image analysis system that straightforwardly guides the clinicians in medical diagnosis. Related tasks such as primitive extraction, position detection, or object recognition all strongly depend on the quality of the segmentation. In this paper, we focus our studies to brain MR imaging where we propose a new automated segmentation method that detects the lesions of MS. Our algorithm 
of segmentation is composed of three stages: segmentation of the brain into regions using the algorithm Fuzzy Particle Swarm Optimization (FPSO) in order to obtain the characterization of the different healthy tissues (White Matter (WM), Grey Matter (GM) and CerebroSpinal Fluid (CSF)) after the extraction of WM. In the second stage, we use a Fuzzy C-Means (FCM) algorithm to eliminate the atypical data of the white matter. Finally, in the third stage, a decision-making system that uses Mamdanitype fuzzy model is employed in order to ascertain whether a given voxel is MS lesion or not.

The paper is organized as follows. First, related works are presented in Section 2. Next, our approach of automatic MS lesion detection and its various steps are highlighted in Section 3. Section 4 examines the results obtained on the MRI images. Finally, a conclusion and perspective work are reported in Section 5 .

\section{RELATED WORK}

A variety of approaches to MS lesion segmentation have been proposed in the literature. Generally speaking, they can be classified into two groups: outlier-based and class-based methods.

In outlier-based methods [1, 11-15] MS lesions are treated and detected as the outliers to the normal brain tissue distribution, which is usually modelled with a Finite Gaussian Mixture (FGM) of CSF, GM and WM classes. Van Leemput et al. [11] pioneered this approach where an (iterative) robustized expectation-maximization like method was promoted such that contextual information were incorporated. Observed intensity values whose Mahalanobis-distances exceed some empirically predefined threshold are marked as lesions. Bricq et al. [16] applied neighborhood information during the inference process using a Hidden Markov Chain model and outliers were extracted using the Trimmed Likelihood Estimator (TLE) [17]. This approach was evaluated on real data including MS lesions using T1 and FLAIR MR images.

Freifeld et al. [18] used a probabilistic model named Constrained Gaussian Mixture Model (CGMM) based on a mixture of multiple gaussian distribution for each brain tissue from T1, T2 and PD modalities. The parameters of this model were estimated using the EM method. MS lesions were recognized as outlier gaussian components and were grouped to form a new class besides other tissues. A probability-based curve evolution technique was utilized to refine the delineation of lesion boundaries. This method is automated and does not need an atlas for parameter initialization. Experimental results on both simulated brain MRI data and real data showed that this method outperformed many state of art approaches, especially in case of very noisy data. To find the list of nearest gaussians for each voxel, the K-Distance Transform (KDT) [19] was used. A rule-based system was designed to distinguish the MS lesion Gaussians from other tissue. Liu et al. [20] introduced a fully automatic method for segmentation of MS lesions from T2-w and FLAIR MR Images using the EM method to separate background, CSF, and $\mathrm{WM}+\mathrm{GM}$. This approach assumed MS lesions as outliers to the brain tissue WM and GM distribution, and the separation was implemented by minimizing a statistically robust L2E measure, defined as the squared difference between the true density and the assumed Gaussian mixture.

The approach proposed by Prastawa et al. [21] combines outlier detection and region partitioning together. The salient point of this approach lies in the way contextual information is incorporated: tissue typing is carried out based on regions (connected groups of voxels) instead of individual voxels. Voxels labeling is conducted through maximizing overall relative entropy or Kullback-Leibler divergence between neighboring regions. Samples with Mahalanobis distance greater than a manually chosen threshold are treated as outliers. Souplet et al. [13, 14] also modelled lesions as an outlier class. In their approach, a segmentation of the brain into CSF, GM and WM was first performed on the T1-weighted (T1w) and T2-weighted (T2w) sequences. Lesions were then detected through a thresholding step on T2w + FLAIR sequences. A similar procedure was adopted in Garcia-Lorenzo et al. [15]. Their solution consists of three steps: (1) robust estimation of normal appearing brain tissue (NABT) parameters, (2) refinement of outlier detection and (3) application of lesion rule. After the NABT distribution is obtained through a robust expectation maximization algorithm, each voxel in the image is labeled as an outlier (candidate for lesions) if the Mahalanobis distance for each class is greater eat than a given $p$-value. The final lesion rule step refines the segmentation result by discriminating White Matter Lesions (WML) from false positives and pruning the latter. Manually chosen thresholds were involved in both the outlier detection and WML separation steps.

Class-based methods [21-26] modeled the lesions as an independent class to be extracted. In Ref. [22], a combination of intensity-based k-nearest neighbor classification (k-NN) and a template-driven segmentation (TDS) was designed to segment different types of brain tissue. Lesions were modeled as one of the expected tissue types, and the class parameters were obtained through a supervised voxel sampling scheme on two randomly selected scans. Since the manual training step is highly datadependent, it is expected to be conducted for each study or data set. A similar approach was proposed in Ref. [12] where the segmentation method determines for each voxel in the image the probability of being part of MS-lesion tissue, and the classification was conducted using K-NN algorithm. Voxel intensities and spatial information were integrated as discriminative features, and voxels were classified based on their proximity to the pre-classified samples in the feature space. It should be noted that manual or semiautomatic training is normally a required step in $\mathrm{k}-\mathrm{NN}$ based methods, and the value of $\mathrm{k}$ (number of classes) has to be determined in advance, either interactively [22] or empirically [12]. Ideally, in order to obtain desired segmentation results, the testing data sets are also expected to be highly similar to the training sets. Outlier-based models [11-15] relax the training requirement while using a thresholding-based technique. Such thresholds, although critical to segmentation quality and system reproducibility, often require some prior information to be set up precisely, which is often difficult to be determined.

Among class-based methods, one distinguishes Atlas-assisted segmentation framework proposed in Refs. [23-25] which makes use of the relatively consistent continuity property and the existing relationship among neighboring anatomical structures within the same group of subjects. Lesions are treated as a subclass within the white matter tissue, and a topology preservation criterion is employed to guarantee strict topological equivalence between the segmented image and the atlas. However, to ensure the strict correspondence between atlas and the patient images, an (augmented) atlas that accurately represents the group subjects is likely to be required for each study. A summary of the aforementioned techniques is highlighted in Table I. 
Table I. Comparaison of MS lesion segmentation methods.

\begin{tabular}{|c|c|c|c|}
\hline Author & Method & Sequences & Evaluation \\
\hline Jain et al. [1] & MSmetrix & $\begin{array}{l}\text { 3D T1-w } \\
\text { 3D FLAIR }\end{array}$ & $\begin{array}{r}\text { Sen }=0.57 \\
\text { Pre }=0.83\end{array}$ \\
\hline Leemput et al. [11] & EM & $\begin{array}{l}\text { T2-w and } \\
\text { PD-w }\end{array}$ & $\mathrm{Si}=0.51$ \\
\hline Anbeek et al. [12] & $\mathrm{kNN}$ & $\begin{array}{l}\text { T1-w, T2-w, } \\
\text { PD-w and } \\
\text { FLAIR }\end{array}$ & $\mathrm{Si}=0.77$ \\
\hline Souplet et al. [13] & EM & $\begin{array}{l}\text { T1-w, T2-w } \\
\text { and FLAIR }\end{array}$ & $\begin{array}{l}\text { Spe }=0.99 \\
\text { Sen }=0.26\end{array}$ \\
\hline Ait-ali et al. [14] & $\begin{array}{l}\text { Robust EM, OD } \\
\text { and MLE }\end{array}$ & $\begin{array}{l}\text { T1-w, T2-w } \\
\text { and PD-w }\end{array}$ & NA \\
\hline $\begin{array}{l}\text { Garcia-Lorenzo } \\
\text { et al. [15] }\end{array}$ & Modified EM & $\begin{array}{l}\text { T1-w, T2-w } \\
\text { and PD-w }\end{array}$ & $\mathrm{Spe}=0.99$ \\
\hline Freifeld et al. [18] & $\begin{array}{l}\text { Constrained } \\
\text { Gaussian } \\
\text { mixture and OD }\end{array}$ & $\begin{array}{l}\text { T1-w, T2-w } \\
\text { PD-w and } \\
\text { FLAIR }\end{array}$ & NA \\
\hline Prastwa et al. [21] & $\begin{array}{l}\text { Bayesian } \\
\quad \text { classification }\end{array}$ & $\begin{array}{l}\text { T1-w, T2-w } \\
\text { and FLAIR }\end{array}$ & $\begin{array}{l}\text { Spe }=0.99 \\
\text { Sen }=0.03\end{array}$ \\
\hline Wu et al. [22] & kNN & $\begin{array}{l}\text { T1-w, T2-w } \\
\text { and PD-w }\end{array}$ & $\begin{array}{l}\text { Sen }=0.70 \\
\text { Spe }=0.98\end{array}$ \\
\hline Zhang et al. [26] & $\mathrm{SWE}+\mathrm{KNN}$ & MS image & $\begin{array}{l}\text { Spe }=0.99 \\
\text { Sen }=0.94\end{array}$ \\
\hline
\end{tabular}

\section{PROPOSED APPROACH}

In our study, we use information from T1-weighted, T2-weighted and proton density-weighted (PD) images. This is motivated by the fact that T1-w, T2-w and PD images contain information about white matter lesions [15]. Therefore, exploring the redundancy and complementarity of such information through appropriate fusion scheme would undoubtedly enhance the efficiency of multiple lesion identification. The overall approach involves several steps. Prior to such processing, an initial registration step that would ease the matching of the voxels of the various image types (T1-w, T2-w, PD) given the differences in both time and modality is required in order to ensure that the same voxel is reproduced in T1-w, T2-w and PD images. Next, a multi-level segmentation based approach is adopted. A generic workflow of the approach is provided in Figure 1. More specifically, the approach makes use of both unsupervised reasoning offered by atwo step classification method as well as an approach that mimics expert reasoning in order to identify whether a potential voxel is a lesion or not. An optimization based approach involves first the identification of WM class at each of the imagery type using a Fuzzy Particle Swarm Optimization (FPSO) algorithm assuming the voxels are WM, GM or CSF as hypothesized in Ref. [15]. The focus on WM is also rooted to related clinical studies, see, e.g., Refs. $[34,35]$ which indicated that the infringement predominantly inflammatory present in the WM is likely in relationship with the mechanisms of degeneration and achievement where the measurement of the load lesional provides insights into the degree of achievement of the WM in the course of the disease. Second, following the argumentation highlighted by AitAli et al. [39], WM tissue is often pervaded by atypical data, which often weakens the detection of lesions. Therefore, discarding the negative effect of atypical data becomes necessarily. For this purpose, an approach based on Fuzzy C-Means algorithm is employed in this paper. Finally, in order to decide whether a given filtered WM voxel is a lesion or not, a fuzzy like reasoning that imitates expert reasoning which gathers global information regarding image contrast as well as the signal type before making such decision. Figure 1 summarizes the processing sequence proposed for the segmentation of MS lesions, while details of the different stages are provided in the subsequent subsections.

\subsection{Segmentation of the Brain Tissues Using Fuzzy Particle Swarm Optimization Algorithm}

The segmentation of the brain tissues into different compartments (white matter (WM), gray matter (GM) and cerebrospinal fluid (CSF)) is a key step in our study. The outcome of this segmentation serves as the basis for implementing lesion-handling based strategies. For this purpose, an optimization based approach using Fuzzy Particle Swarm Optimization algorithm has been adopted in our approach. This is motivated by its simplicity, ability to deal with high dimension dataset, as well as its proven efficiency in similar other segmentation tasks as pointed out in Refs. [36, 37]. The application of Fuzzy Particle Swarm Optimization (FPSO) approach for clustering in our case yields three distinct classes corresponding to white matter (WM), gray matter (GM) and cerebrospinal fluid (CSF).

\subsubsection{Particle Swarm Optimization (PSO)}

The PSO is a population-based stochastic method inspired by bird flocking and fish schooling to find optimal or near-optimal solutions. It was first introduced in 1995 by social-psychologist Eberhart and electrical engineer Kennedy [38], and nowadays, has been used used in many fields involving large scale optimization problems.

More formally, PSO starts starts with a population of particles. Each particle $i$ consists of potential solutions called positions $X$, and velocities $V$ and maintains the following information [30]:

- $x_{i}$, the current position of the particle.

- $v_{i}$, the current velocity of the particle.

- $y_{i}$, the personal best position of the particle (pbest); the best position visited so far by the particle.

- $\hat{y}$, the global best position of the swarm (gbest); the best position visited so far by the entire swarm

In each iteration $t$, the performance of each particle $i$ is measured using a predefined fitness function $f$. The personal best position (pbest) is obtained as follows [30]:

$$
y_{i}(t+1)=\left\{\begin{array}{l}
y_{i}(t) \text { if } f\left(x_{i}(t+1)\right) \geq f\left(y_{i}(t)\right) \\
x_{i}(t+1) \text { if } f\left(x_{i}(t+1)\right)<f\left(y_{i}(t)\right)
\end{array}\right.
$$

There are two different topologies of PSO algorithm to find best solutions: global and local topologies. In global topology, the position of each particle is affected by the best-fitness particles of the whole swarm in the search space while each particle is influenced by the best-fitness particles of its neighbors in the local topology. In this study we use the global topology whose global best position $\hat{y}$ is obtained as follows [30]

$$
\begin{aligned}
\hat{y}(t) & \in\left\{y_{1}, y_{2}, \ldots, y_{p}\right\} \\
= & \min \left\{f\left(y_{1}(t)\right), f\left(y_{2}(t)\right), \ldots, f\left(y_{p}(t)\right)\right\}
\end{aligned}
$$

The particle's velocity and position are updated as follows:

$$
\begin{aligned}
v_{i}(t+1)= & w v_{i}(t)+c_{1} r_{1}(t)\left(y_{i}(t)-x_{i}(t)\right) \\
& +c_{2} r_{2}(t)\left(\hat{y}(t)-x_{i}(t)\right) ; \quad i=1,2, \ldots, p
\end{aligned}
$$




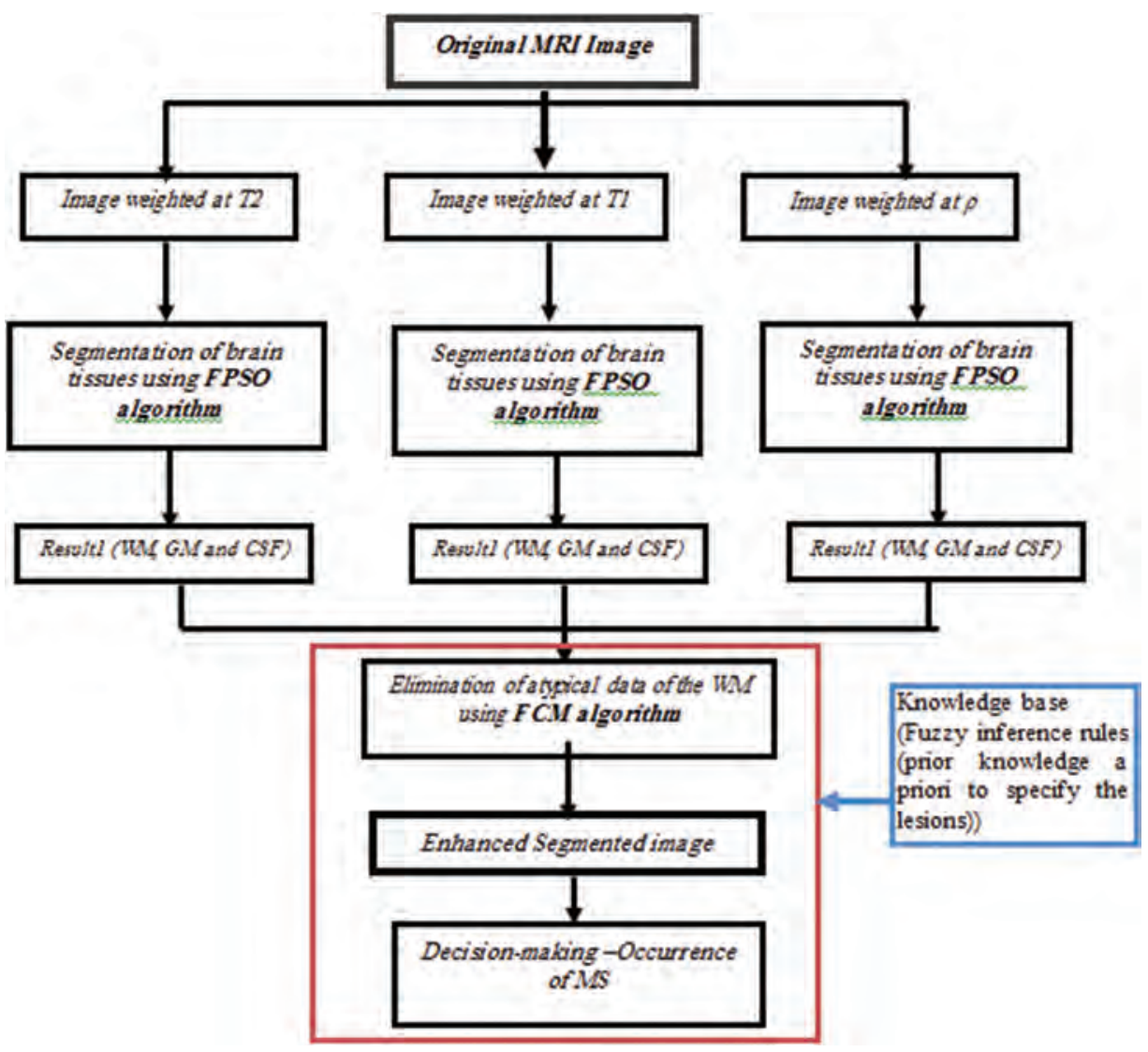

Fig. 1. Details of the proposed method.

$$
x_{i}(t+1)=x_{i}(t)+v_{i}(t+1) ; \quad i=1,2, \ldots, p
$$

where, $w$ is inertia weight that controls the impact of previous velocity of particle on its current one, $c 1$ and $c 2$ are positive constants, called acceleration coefficients which control the influence of pbest and gbest on the search process, $p$ is the number of particles in the swarm, and $r 1$ and $r 2$ are random values in range $[0,1]$. The PSO algorithm is repeated until a specified number of iterations has been exceeded or the velocity changes are close to zero.

Particle swarm optimization (PSO) is a population-based optimization tool, which could be implemented and applied easily to solve various function optimization problems, or the problems that can be transformed to function optimization problems [40]. However, the PSO algorithm suffers a serious problem that all particles are prone to be trapped into the local minimum in the later phase of convergence. The optimal value found is often a local minimum instead of a global minimum [42]. Pang et al. [32] proposed a version of particle swarm optimization for TSP called fuzzy particle swarm optimization (FPSO).

\subsubsection{Fuzzy Particle Swarm Optimization for Fuzzy Clustering}

Aiming at solving the shortcoming of the PSO algorithm, many variations, such as Fuzzy PSO [42]. The FPSO algorithm was initially proposed by Pang et al. [32] to solve Traveling Salesman Problem (TSP). In which routine the position and velocity of particles redefined to symbolize the fuzzy family member between variables. In this sub-section, we present the FPSO algorithm for fuzzy clustering problem [40].

In FPSO algorithm, $X$, the position of particle, represents the fuzzy memberships of pixels $\left\{o_{1}, o_{2}, \ldots, o_{N}\right\}$, to set of cluster centers $\left\{Z_{1}, Z_{2}, \ldots, Z_{c}\right\}, X$ can be expressed as follows:

$$
X=\left[\begin{array}{ccc}
u_{11} & \cdots & u_{1 c} \\
\vdots & \ddots & \vdots \\
u_{N 1} & \cdots & u_{N c}
\end{array}\right]
$$

$u_{i j}$ is the membership function of the $i$-th pixel to the $j$-th cluster with constraints stated in Eq. (11). Therefore, the position matrix of each particle is similar to the fuzzy matrix $U$ in FCM algorithm. Also, the velocity of each particle is stated using a matrix $V$ with the size $N$ rows and $c$ columns. Equations (6) and (7) are used to update the positions and velocities [40].

$$
\begin{gathered}
V(t+1)=\omega \otimes V(t) \oplus\left(c_{1} r_{1}\right) \otimes\left(y_{i}(t) \ominus X(t)\right) \\
\oplus\left(c_{2} r_{2}\right) \otimes(\hat{y}(t) \ominus X(t)) \\
X(t+1)=X(t) \oplus V(t+1)
\end{gathered}
$$

where the symbols $\oplus$ and $\ominus$ denote the addition and subtraction between matrices respectively. The symbol $\otimes$ denote a multiplication of all elements in the matrix by a real number.

After updating the position matrix $X$, it may violate the constraints given in Eq. (11). So it is necessary to normalize the 


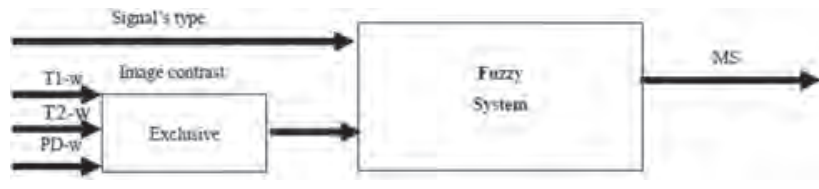

Fig. 2. Diagram of fuzzy system of the MS disease.

position matrix $X$ [40]. First, all negative elements in matrix $X$ are set to zero. If all elements in a row of the matrix $X$ are zero, they need to be re-evaluated using series of random numbers within the interval $[0,1]$ and then the matrix undergoes the following transformation without violating the constraints [40]:

$$
X_{\text {normal }}=\left[\begin{array}{ccc}
u_{11} / \sum_{j=1}^{c} u_{1 j} & \cdots & u_{1 c} / \sum_{j=1}^{c} u_{1 j} \\
\vdots & \ddots & \vdots \\
u_{N 1} / \sum_{j=1}^{c} u_{N j} & \cdots & u_{N c} / \sum_{j=1}^{c} u_{N j}
\end{array}\right]
$$

Similarly to other evolutionary algorithms, in FPSO, we also need a function for evaluating the generalized solutions called fitness function. In this paper, Eq. (9) is used for evaluating the solutions.

$$
f(X)=K / J_{m}
$$

Therein $K$ is a constant and $J_{m}$ is the objective function of FCM algorithm. The FPSO algorithm for fuzzy clustering problem is therefore summarized in Algorithm 1 below.

\section{Algorithm 1.}

input original image.

1. Initialize the parameters including population size

$P, c 1, c 2, w$, and the maximum iterative count.

2. Create a swarm with $P$ particles

( $X$, pbest, gbest and $V$ are $n * c$ matrices).

3. Initialize $X, V$, pbest for each particle and gbest for the swarm.

4. Calculate the cluster centers for each particle using Eq. (13).

5. Calculate the fitness value of each particle using Eq. (9).

6. Calculate pbest for each particle using Eq. (1).

7. Calculate gbest for the swarm using Eq. (2).

8. Update the velocity matrix for each particle using Eq. (6).

9. Update the position matrix for each particle using Eq. (7).

10. If terminating condition is not met, go to step 4.

output segmented image

The termination condition in proposed method is the maximum number of iterations or no improvement in gbest in a number of iterations.

Table II. Rules' base in the form of a matrix.

\begin{tabular}{lclc}
\hline & T1-w & T2-w & PD-w \\
\hline Hypersignal & Low/Normal & High & High \\
Hopersignal & Low & High & High \\
$\begin{array}{l}\text { Hypersignal after injection } \\
\text { of gadolinium }\end{array}$ & Normal & High & High \\
\hline
\end{tabular}

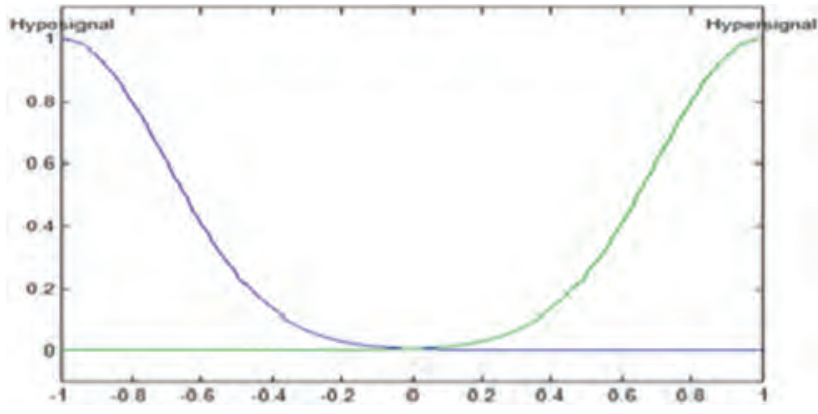

Fig. 3. Fuzzy repartition of input variable of signal's type.

\subsection{Segmentation of the White Matter Using Fuzzy C-Means Algorithm}

The next stage in our methodology consists in eliminating the atypical data in previously identified WM voxels in order to highlight the different MS lesions. This is because the lesions of the multiple sclerosis are not well contrasted due to the partial volume in the surrounding tissues, which renders their segmentation rather a difficult task. Motivated by the lack of a fully comprehensive labeled database as reported in Ref. [28] a non-supervised like strategy based on fuzzy c-means algorithm has been advocated. This is backed by its reported success in image analysis and medical diagnosis including magnetic imaging regardless of the modality and the type of acquisition (mono or multimodal) $[27,29,31,33]$ its reduced complexity, easy implementation (especially for large and high dimension dataset) [31].

\subsubsection{Fuzzy C-Means Algorithm}

FCM is a fuzzy clustering method based on the minimization of a quadratic criterion where clusters are represented by their respective centers [27]. More specifically, in our context, given a grey-level image $X=x_{1}, x_{2}, \ldots, x_{N}$ where $x_{i}$ corresponds to the grey-level value of the ith pixel and $N$ is the total number of pixels of the image, the FCM algorithm allows us to partition the pixels of $X$ into $C$ classes (here $C=3$ ) pertaining to WM, GM and CSF by calculating the centers $c_{j}(j=1, C)$ of $j$ th class and the membership matrix $(U)$, by minimizing an objective function $J$.

$$
J=\sum_{i=1}^{C} \sum_{j=1}^{N}\left(u_{i j}\right)^{m} d\left(x_{j}, c_{i}\right)^{2}
$$

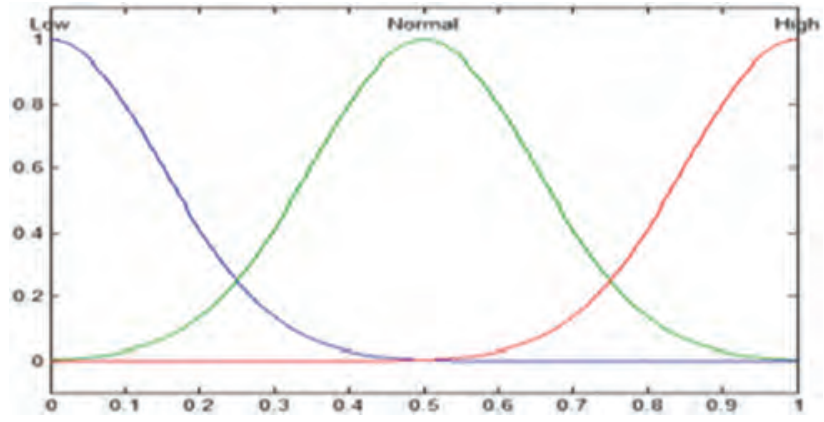

Fig. 4. Fuzzy repartition of the output variable giving the decision of the MS disease. 


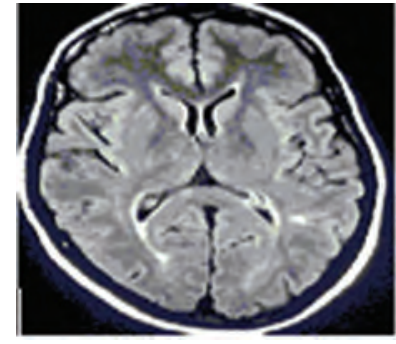

T1 MR Image

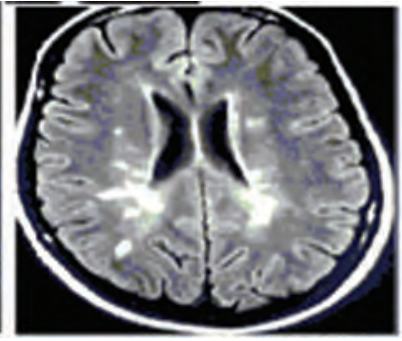

T2 MR Image

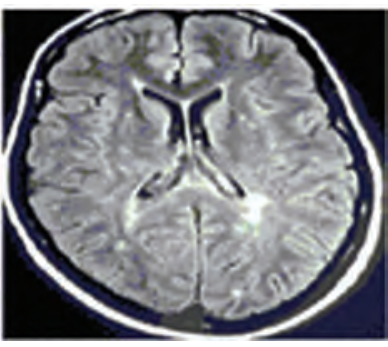

PD MR Image

Fig. 5. Original images

Under constraints:

$$
\begin{gathered}
\forall j \in[1, N]: \sum_{i=1}^{C} u_{i j}=1 ; \\
\forall i \in[1, C], \quad \forall j \in[1, N] u_{i j} \in[0,1]
\end{gathered}
$$

Where: $U=\left[u_{i j}\right]_{C * N}$ is the membership function matrix, $d\left(x_{j}, c_{i}\right)$ is the Euclidean distance from the $j$ th pixel to $i$ th class, $C$ is the number of clusters, $N$ is the number of data, $m$ is the degree of fuzziness $m>1$.

The membership functions and cluster centers are updated by Eqs. (3) and (4) respectively.

$$
u_{i j}^{(k)} \leftarrow\left[\sum_{I=1}^{C}\left(\frac{d\left(x_{j}, c_{j}^{(k-1)}\right)}{d\left(x_{j}, c_{I}^{(k-1)}\right)}\right)^{2 /(m-1)}\right]^{-1}
$$

$$
c_{i}^{(k)} \leftarrow \frac{\sum_{j=1}^{N}\left(u_{i j}^{(k)}\right)^{m} \cdot x_{j}}{\sum_{j=1}^{N}\left(u_{i j}^{(k)}\right)^{m}}
$$

The solution of the optimization problem (1-2) is obtained using Lagrange multiplier approach [27] through an iterative based method whose algorithmic detail is summarized in Algorithm 2. Especially, both centers of classes and membership degrees cannot be found directly at the same time, so an alternating procedure is used. Firstly, the prototypes of classes are fixed arbitrary to find the membership degrees, secondly, the membership degrees are fixed to find the corrected prototype centers. These two steps are alternatively repeated until convergence is attained. The number of tissue classes was assumed to be three, corresponding to gray matter $(\mathrm{GM})$, white matter (WM) and cerebrospinal fluid (CSF) tissue classes. (a)

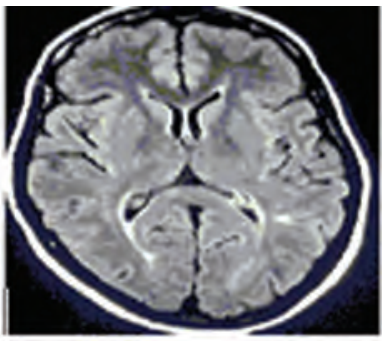

(b)

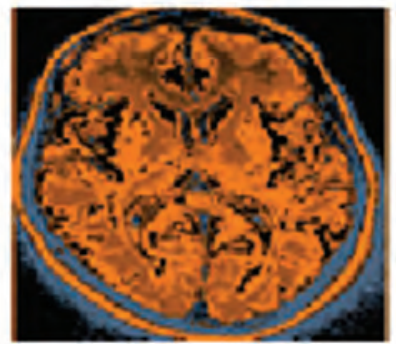

(c)

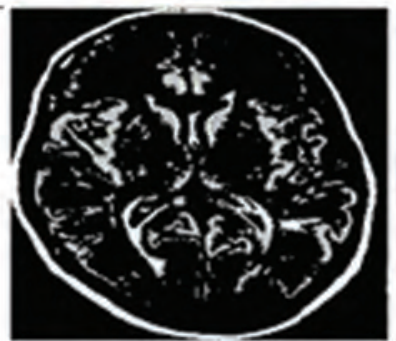

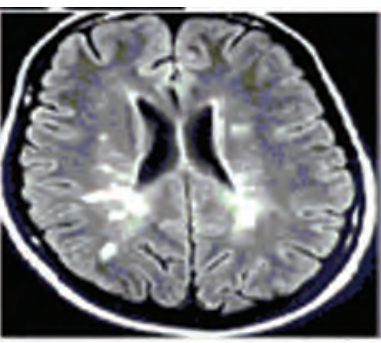
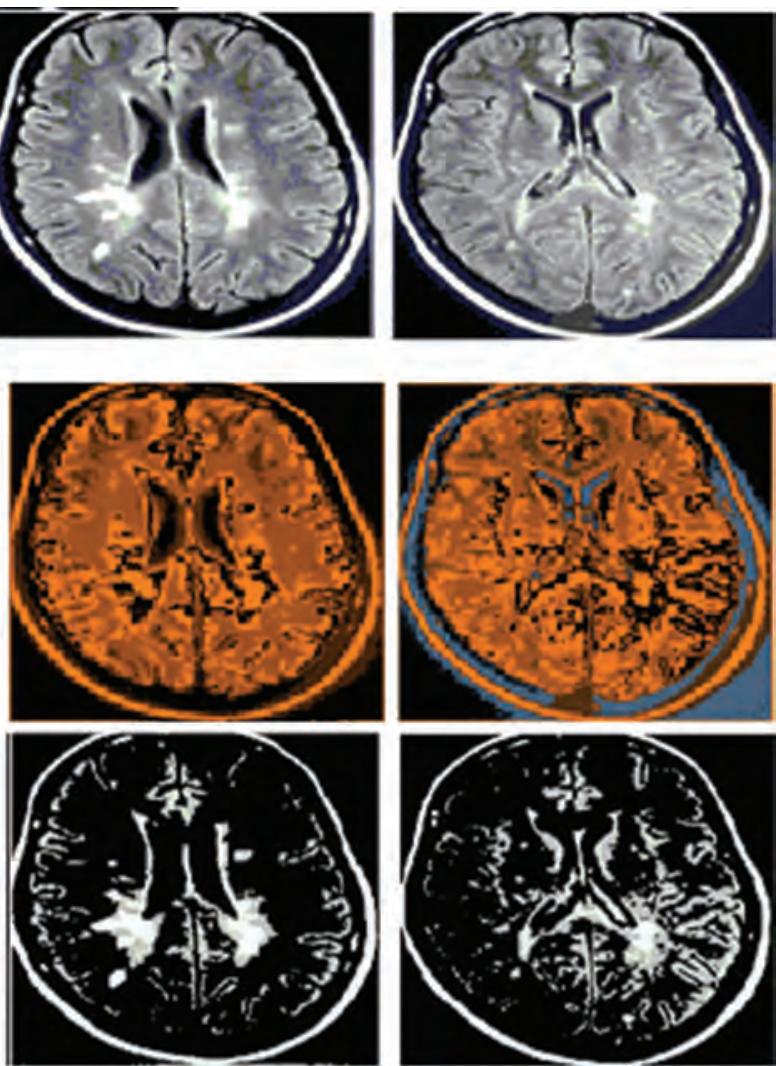

Fig. 6. (a) Is the original images; (b) is the FPSO segmentation results; (c) is the white matter (WM) extraction. 
Table III. Comparison of the results obtained by FPSO algorithm.

\begin{tabular}{cccc}
\hline & CSF & WM & GM \\
\hline T1-w & & & \\
Si & 0.83 & 0.93 & 0.86 \\
Ovrl & 0.67 & 0.90 & 0.83 \\
Sen & 0.73 & 0.96 & 0.90 \\
Spc & 0.79 & 0.97 & 0.92 \\
T2-w & & & \\
Si & 0.94 & 0.96 & 0.95 \\
Ovrl & 0.90 & 0.93 & 0.92 \\
Sen & 0.91 & 0.95 & 0.94 \\
Spc & 0.95 & 0.97 & 0.95 \\
PD-w & & & 0.80 \\
Si & 0.78 & 0.83 & 0.70 \\
Ovrl & 0.58 & 0.76 & 0.74 \\
Sen & 0.65 & 0.81 & 0.87 \\
Spc & 0.89 & 0.87 & \\
\hline
\end{tabular}

Algorithm 2.

Require. Set value Set values for the number of clusters $C$, the degree of fuzziness $m>1$ and the error $\epsilon$.

1. initialize randomly the centers of clusters $c_{i}^{(0)}$.

2. $k \leftarrow 1$.

3. repeat

4. Update the membership function $U^{(k)}$ according to Eq. (12)

5. Update the centers $c_{i}^{(k)}$ according to Eq. (13)

6. $k \leftarrow k+1$.

7. until $\left\|C_{i}^{(k)}-C_{i}^{(k+1)}\right\| \leqslant \epsilon$

8. return $c_{j}$ the centers of clusters and the membership degrees $u_{i j}$.

\subsection{Decision-Making}

The last step determines whether a given WM voxel is MS lesion or not. For this purpose, a Mamdani-type fuzzy inference system has been adopted. In the latter, (global) information about the image contrast and signal's type are used as global variables. The outcome corresponds to the extent to which the MS attribute is persistent in the underlying WM voxel. Especially, the weighted images in T2 and PD underline the myelin component in the lesions characterized by the edemas with hyper-intense appearance in comparison to the white matter. Furthermore, T1-w underlines the irreversible destruction of the tissues with the appearance in the white matter of persistent "black holes" (Hypo-signal) [39].

In this study, experts' knowledge was used for the construction of fuzzy rule base. It would not be possible to derive directly all rules from the experts due to the combination of the input variables and their linguistic values. To develop this rule base it is always advised to hire a knowledge engineer who elicits knowledge from the domain experts and transfer those elicited knowledge into rule base. In this paper, the expert have been defined seventeen fuzzy rules by observation on the data. Ten of the rules keep the lesion and rest removes the FPs (False Positive). An instance of fuzzy rules is described below.

1. If [(the image contrast is T1-w active) AND (the signal is hyperintense)] then (MS is low).

2. If [(the image contrast is T1-w active) AND (the signal is hyperintense)] then (MS is normal).

3. If [(the image contrast is $\mathrm{T} 2-\mathrm{w}$ active) AND (the signal is hyperintense)] then (MS is high)

4. If [(the image contrast is PD-w active) AND (the signal is hyperintense)] then (MS is high).

5. If [(the image contrast is T1-w active) AND (the signal is hypointense)] then (MS is low).

6. If [(the image contrast is T2-w active) AND (the signal is hypointense)] then (MS is high).

7. If [(the image contrast is PD-w active) AND (the signal is hypointense)] then (MS is high).

8. If [(the image contrast is T1-w active) AND (the signal is hyperintense after injection of gadolinium)] then (MS is normal). 9. If [(the image contrast is $\mathrm{T} 2-\mathrm{w}$ active) AND (the signal is hyperintense after injection of gadolinium)] then (MS is high). 10. If [(the image contrast is PD-w active) AND (the signal is hyperintense after injection of gadolinium)] Then (MS is high).

The quantification of image contrast, signal type and the MS disease is described in the following:

For the fuzzification of signal's type, we choose two fuzzy intervals and Gaussian type membership function. Figure 3 shows the fuzzy repartition of the input variable of signal's type.

For the output variable, we choose three fuzzy intervals and Gaussian membership functions, which define predicates: low, normal and high of the MS disease in comparison to the white matter. Figure 4 shows the fuzzy repartition of the output variable of the decision of the MS disease.

The selected inference method is Mamdani's method. Consequently, the operator is realized by the calculation of the minimum, whiles the operator OR is realized by the calculation of the maximum. The defuzzification step is done using the method of calculating the center of attraction.

\section{RESULTS AND DISCUSSION}

\subsection{Dataset}

Framework using real dataset. The dataset was provided to us as part of a collaboration agreement between LSI laboratory

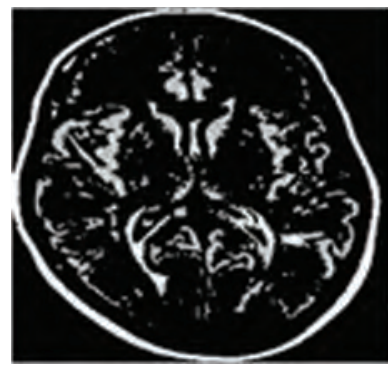

(a) (WM) at T1

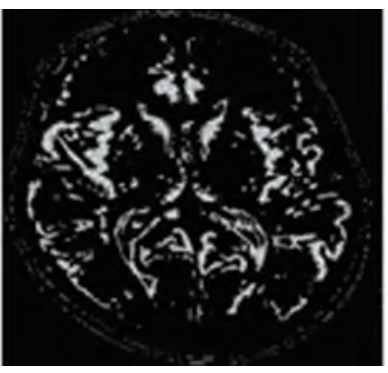

(b) WM

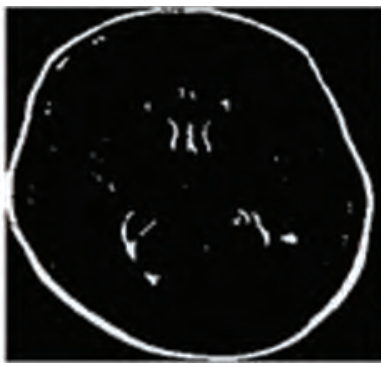

(c) Atypical Data

Fig. 7. WM of the image T1 segmented by FCM. 


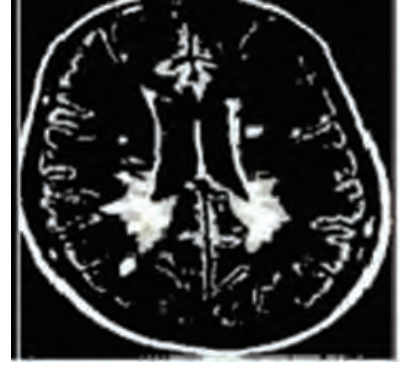

(a) (WM) at T2

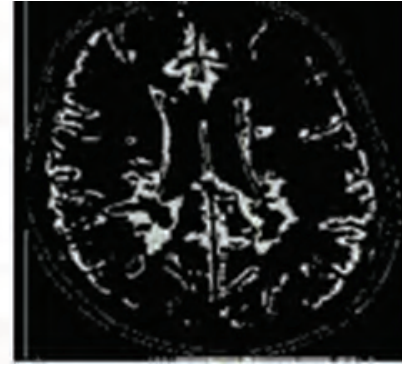

(b) WM

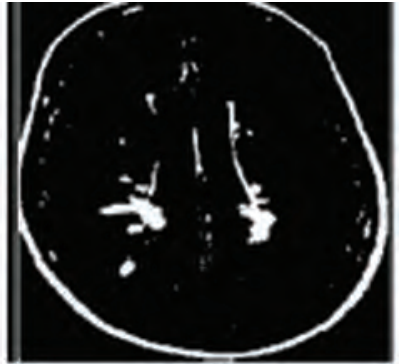

(c) Atypical Data

Fig. 8. WM of the image T2 segmented by FCM.

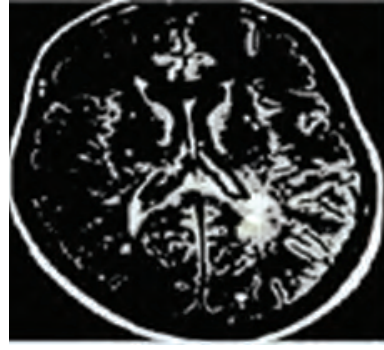

(a) (WM) at PD

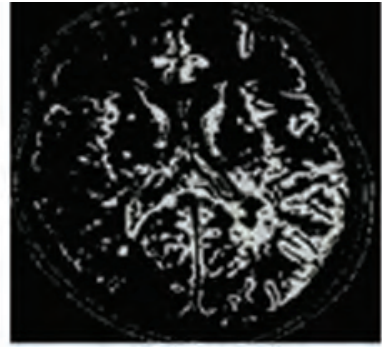

(b) WM Data

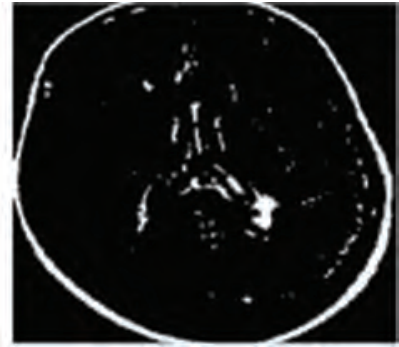

(c) Atypical

Fig. 9. WM of the image PD segmented by FCM

(Laboratory Intelligent Systems: image and signal team) Ferhat Abbas University of Setif and LAMIH UMR CNRS 8201 (Laboratory of Industrial and Human Automation control, Mechanical engineering and Computer Science) University of Valenciennes. The various images weighted at T1, T2 and PD corresponding to relatively older patients and the images are in the form of DICOM (Digital Imaging and Communications in Medecine). The images were already preprocessed and spatially normalized.

\subsection{Computational Requirement}

The proposed algorithm was implemented in NetBeans IDE 8.2 and run on a laptop with $2.40 \mathrm{GHz} \operatorname{Intel}(\mathrm{R})$ Core (TM) i54210U CPU and 4 GB RAM. The operating system was 64-bit Windows 8.1.

Table IV. Comparison of the results obtained by FCM algorithm.

\begin{tabular}{lcc}
\hline & Atypical data & WM \\
\hline T1-w & & \\
Si & 0.92 & 0.90 \\
Ovrl & 0.93 & 0.87 \\
Sen & 0.90 & 0.89 \\
Spc & 0.91 & 0.90 \\
T2-w & & \\
Si & 0.97 & 0.96 \\
Ovrl & 0.94 & 0.93 \\
Sen & 0.93 & 0.90 \\
Spc & 0.96 & 0.94 \\
PD-w & & \\
Si & 0.95 & 0.91 \\
Ovrl & 0.93 & 0.81 \\
Sen & 0.89 & 0.88 \\
Spc & 0.91 & 0.90 \\
\hline
\end{tabular}

To compare the performance of these images, we compute different coefficients reflecting how well two segmented volumes match. Four measures are used as follows [43]:

$$
\begin{gathered}
\text { Overlap }(\mathrm{Ovrl})=\frac{\mathrm{TP}}{\mathrm{TP}+\mathrm{FN}+\mathrm{FP}} \\
\text { Similarity }(\mathrm{Si})=\frac{2 \cdot \mathrm{TP}}{2 \cdot \mathrm{TP}+\mathrm{FN}+\mathrm{FP}} \\
\text { Sensitivity }(\mathrm{Sen})=\frac{\mathrm{TP}}{\mathrm{TP}+\mathrm{FN}} \\
\text { Specificity }(\mathrm{Spc})=\frac{\mathrm{TN}}{\mathrm{TN}+\mathrm{FP}}
\end{gathered}
$$

Where TP and FP stand for true positive and false positive, which were defined as the number of voxels correctly and incorrectly classified as brain tissue by the automated algorithm. TN and FN stand for true negative and false negative, which were defined as the number of voxels correctly and incorrectly classified as non-brain tissue by the automated algorithm.

\subsection{Analysis of the Results}

The various images weighted at $\mathrm{T} 1, \mathrm{~T} 2$ and $\mathrm{PD}$ corresponding to relatively older patients and the images are in the form of DICOM (Digital Imaging and Communications in Medecine). The brain segmentation was successfully applied on some real images shown in Figure 5.

Table V. Result of the defuzzification of the atypique data of the different sequences.

\begin{tabular}{cccc}
\hline & T1-w (\%) & T2-w (\%) & PD-w (\%) \\
\hline MS & 48.76 & 56.79 & 49.56 \\
\hline
\end{tabular}




\subsubsection{Step 1: Segmentation of Tissues (WM,} GM, and CSF) by FPSO

Figure 6 show the segmentation by the FPSO algorithm for the T1-w, T2-w and PD weighted images in order to obtain a characterization of the different healthy tissues White matter, Grey matter and cerebrospinal fluid (see (b)) and afer a segmentation by the FPSO algorithm, we extrated the WM (see (c)).
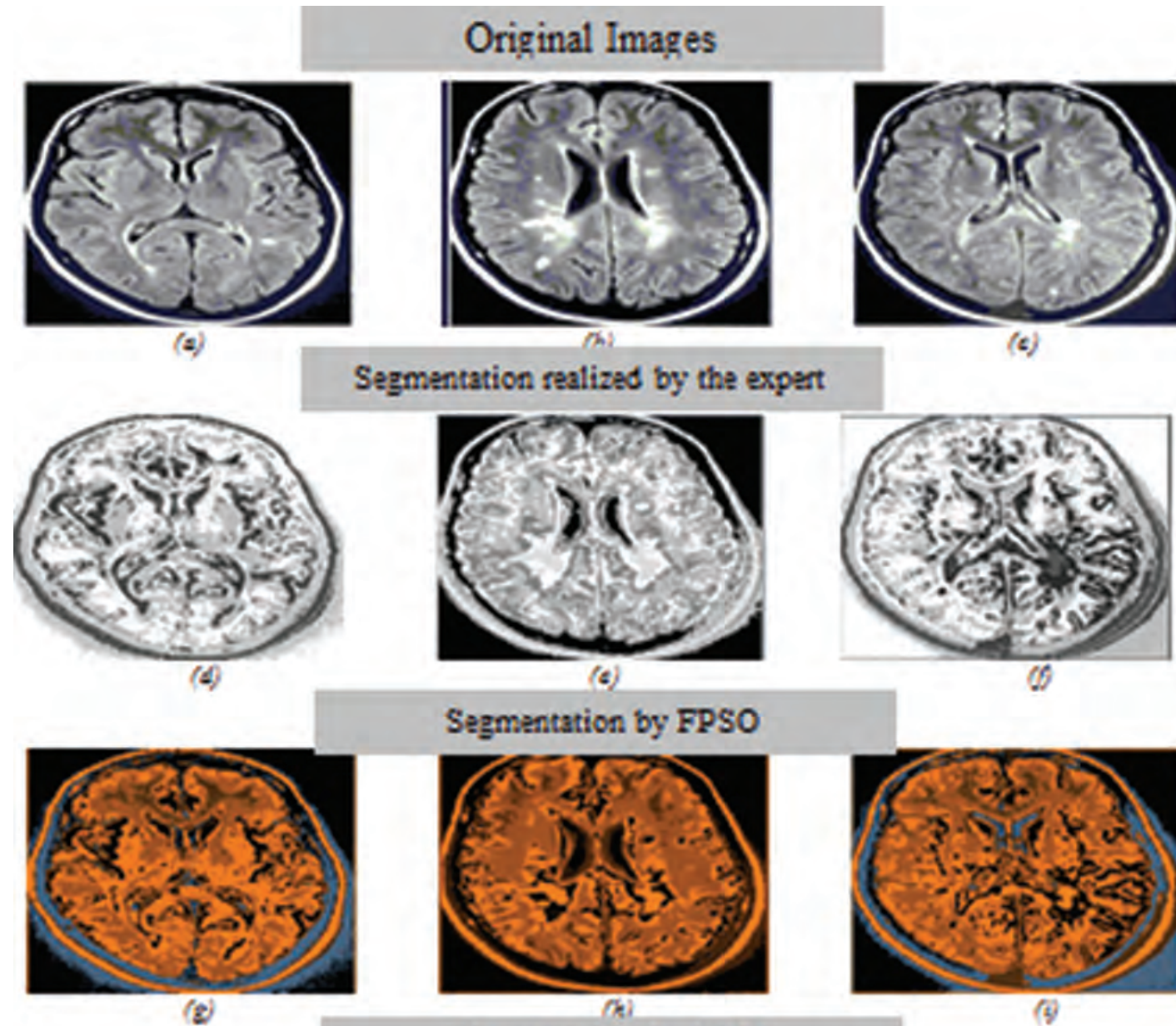

\section{Segmentation by FCM}
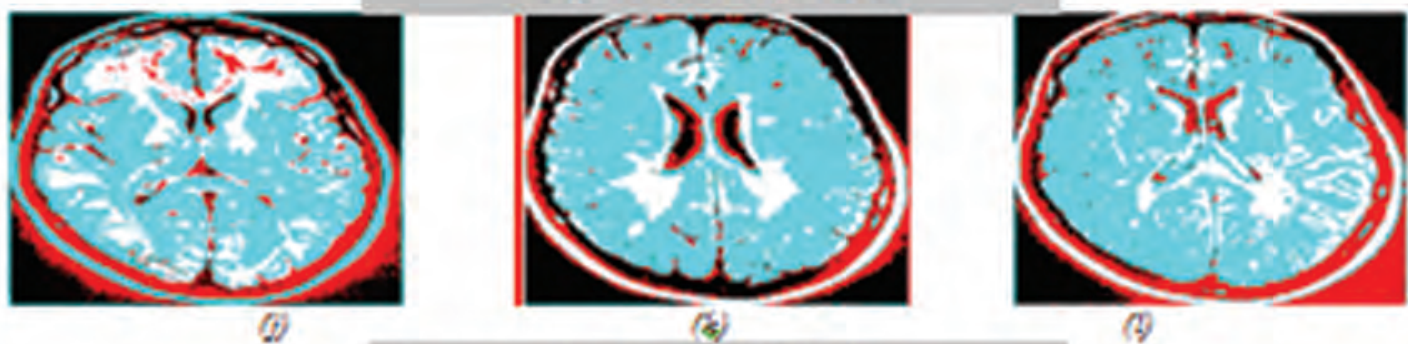

(4)

Segmentation by FPSOFCM
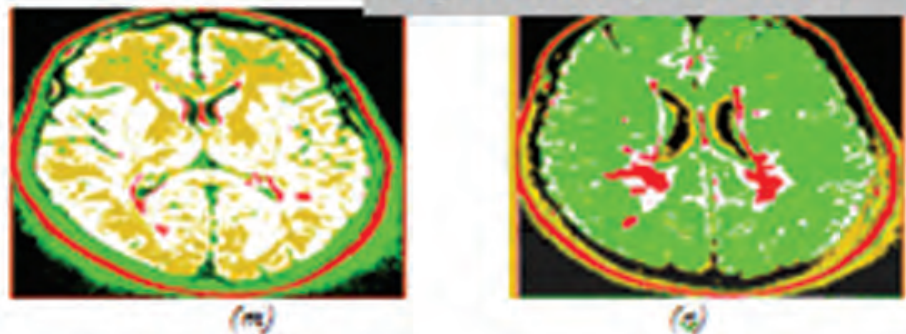

(e)

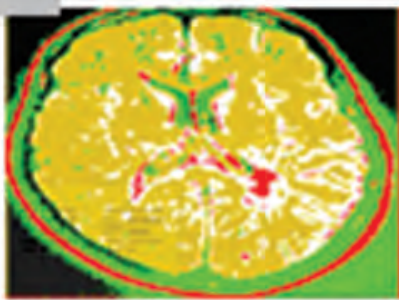

(e)

Fig. 10. Results of segmentation 
The comparative results are presented in Table III above.

The results in Table III show a considerable improvement for all tissues using T1-w than T2-w and PD-w.

\subsubsection{Step 2: Segmentation of the White Matter by FCM}

The use of FCM allows us to eliminate the atypical data of the white matter for each image (T1, T2, PD) as exhibited in Figures 7-9 for images T1, T2 and PD, respectively.

Table IV summarizes the segmentation outcome by FCM.

The results obtained by FCM are very satisfactory and well confirm the validity of the algorithm, its ease of implementation gives us a substantial advantage. We have made an improvement in optimizing the white matter and atypical localization data.

\subsubsection{Step 3: Decision-Making}

The implementation of the Mamdani fuzzy inference system makes use of min operator for AND connective and max for OR connectives. The result of the implementation is shown in Table V.

The decision-making depends always on the expertise, the patient suffers from the multiple sclerosis and the MS lesions are detected in all the sequences by a normal or a high characterization.

\subsection{Experimental Results}

Figure 10 shows the results obtained after segmentation of the different brain tissues of the images (a), (b) and (c) weighted at T1-w, T2-w and PD-w on axial planes. The images (d), (e), (f), (g), (h), (i), (j), (k), (l), (m), (n) and (o) are the results of segmentation realized by the expert, FPSO, FCM and FPSOFCM successively.

The results of each stage of the segmentation are presented on a sectional level (Fig. 5) in which the localization allows distinguishing three separated classes of the tissue:

- GM (Pallidum, Putamen, caudate nucleus, thalamus and cortex).

-WM (brain parenchyma).

- CSF (subarachnoid space, lateral ventricles and V3).

The interpretation of our results is done by an expert (hospital center of Ain Naadja Algiers) on real images. All brain lesions were identified by experienced MS neurologists. By analyzing the images of Figure 10, the expert has established the following statement:

- Image (f): The interpretation of the classes is totally improved in relation to (FPSO, FCM), we notice the distinction between the 03 classes of the brain and the class of the pathology SEP.

- Image (g): The detection of the pathology is indicated according to the expert but the details are not well expressed.

- Image (k): The FCM does not bring much compared to the FPSO.

Table VI. Comparison of the results obtained by different algorithms.

\begin{tabular}{lcccc}
\hline & GM $(\%)$ & CSF $(\%)$ & WM(\%) & MS(\%) \\
\hline FPSO & 80.2 & 64 & 85 & 70 \\
FCM & 71 & 55.9 & 79.6 & 74 \\
FPSOFCM & 88.7 & 66 & 89.7 & 96.4 \\
\hline
\end{tabular}

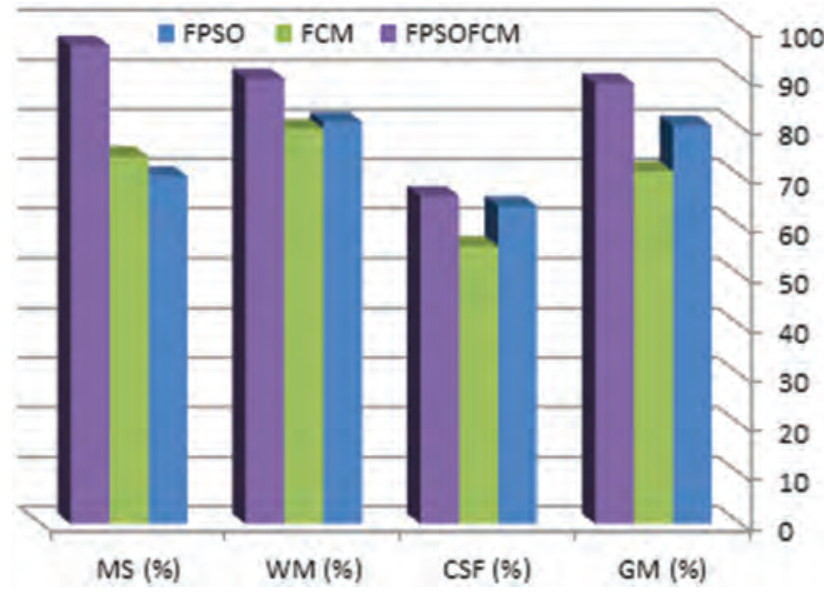

Fig. 11. Performance measures of the results obtained by different algorithms.

- Image (1): FCM is unsuitable in this segmentation in relation to the image (o).

- Image (o): the FPSOFCM brings a great performance to the segmentation for the three classes and especially for the fourth one which is the pathology that specifies well the size and the details about this later.

Next, we compared in Table VI the segmentation of T2-w RMI performed by the expert and that achieved using our automated approach for a given time of acquisition.

Table VI and Figure 11 summarize the results of MS lesion detection algorithms reviewed in terms of reproducibility and agreement with the experts. The results highlighted figure underline the advantages of the proposed approach (FPSOFCM) in comparison to the segmentation by FPSO and FCM for all tissues CSF, WM, GM and MS lesions. We have shown a novel high performance method for the segmentation of abnormal anatomy in MRI data, such as MS lesions.

Next, we also investigated the results using the Receiver Operating Characteristics (ROC) curve. The latter is a pixel based

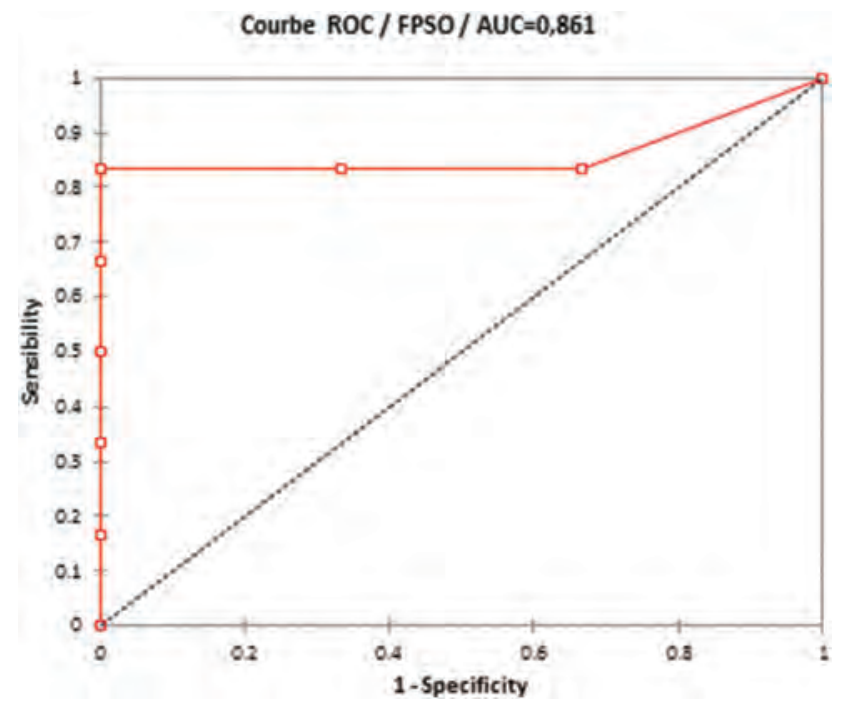

Fig. 12. The curve ROC of a MS by FPSO algorithm. 


\section{Courbe ROC / FCM / AUC $=0,889$}

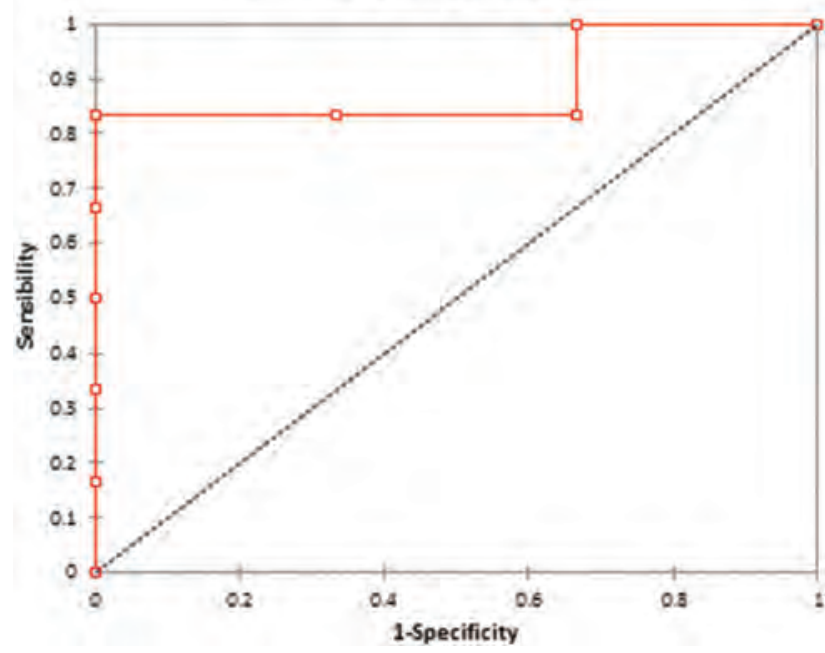

Fig. 13. The curve ROC of a MS by FCM algorithm.

standard measure used to compare the ground truth image and output image of segmentation method based on the use of confusion matrix [41]. Factors involved in the confusion matrix generation are true positives (TP) (MS patient is correctly identified as MS), false positive (FP) (Healthy case is incorrectly identified as MS), the true negative (TN) means healthy people were correctly identified as healthy, and the false negative (FN) means MS patients incorrectly identified as healthy [26]. Sensitivity and 1-specificity are two measures required for plotting the ROC curve. The receiver operating characteristic (ROC) curve and the area under the curve (AUC) parameter of FPSO, FCM and FPSOFCM for the MS are highlighted in Figures 12-14.

The ROC curves for the three methods are shown in Figures 12-14. It is obviously observed that the ROC curve obtained by FPSO and FCM algorithms is much lower than that obtained by the proposed approach (FPSOFCM). Therefore, this comparison reveals that the proposed algorithm (FPSOFCM)

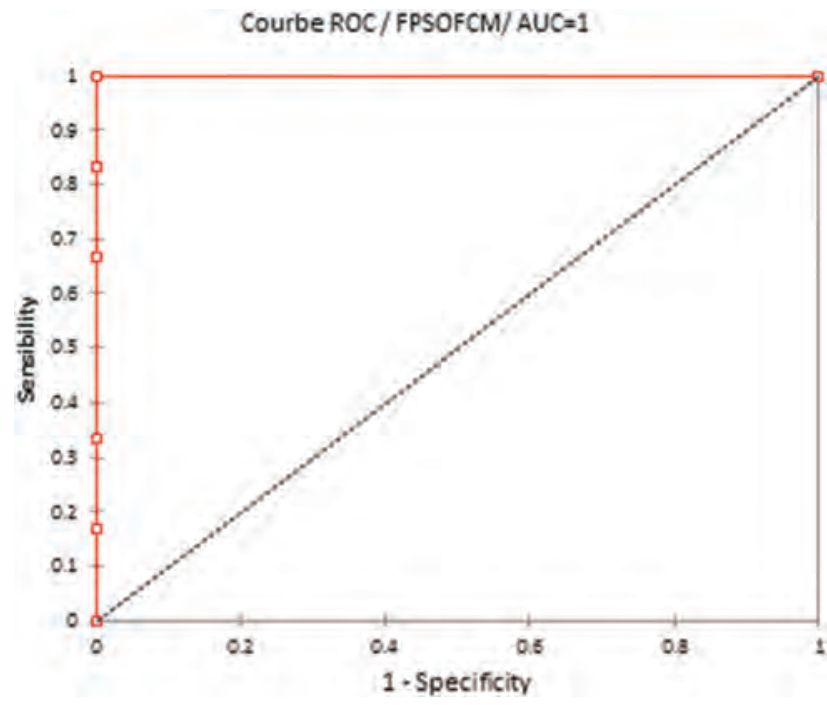

Fig. 14. The curve ROC of a MS by FPSOFCM algorithm. provides a better performance in terms of identifying more true positives than FPSO and FCM algorithms.

\section{CONCLUSION}

The goal of the research presented in this article was to propose a new automatic approach of segmentation of the MS lesions' images. We have firstly split up the process of automatic segmentation of the MS lesions into three fundamental stages:

Firstly, we segmented the brain into regions by using the algorithm Fuzzy Particle Swarm Optimization (FPSO) in order to obtain the characterization of the different healthy tissues (White matter, Grey matter and cerebrospinal fluid (CSF) after the extraction of white matter (WM). Secondly, we eliminated of the atypical data of the white matter by the algorithm Fuzzy C-Means (FCM). Finally, in the framework of our application on the MS disease, we used a Mamdani-type fuzzy model to make decision of the MS disease. We presented the results of our work consisting in the use of an algorithm for the segmentation if medical images in order to improve the quality of the MS lesions' detection. The good quality of our solutions depends on the fact that:

It is a method totally automatic due to the modeling of the prior knowledge of the neuroradiology experts. The fuzzy theory is important for modeling the human knowledge using the mathematical functions and to solve the effect of the partial volume of the MRI.

It satisfies the application's constraints due to the automaticity and the different final results which may be provided by the fuzzy $3 \mathrm{D}$ reconstruction

Its performance is better than the performance of the supervised method.

It is a system based on fuzzy and optimization theory.

It is efficient on 2 types of tissues at least.

\section{References and Notes}

1. Jain, S., Sima, D.M., Ribbens, A., Cambron, M., Maertens, A., Hecke, W.V., Mey, J.D., Barkhof, F., Steenwijk, M.D., Daams, M., Maes, F., Huffel, S.V., Vrenken, H. and Smeets, D., 2015. Automatic segmentation and volumetry of multiple sclerosis brain lesions from MR images. Neurolmage: Clinical 8.

2. lacobaeus, E., lacobaeus, E., Sugars, R.V., Andren, A.T., Alm, J.J., Qian, H., Frantzen, J., Newcombe, J., Alkass, K., Druid, H., Bottai, M., Roytta, M., Le Blanc, K., 2017. Dynamic changes in brain mesenchymal perivascular cells associate with multiple sclerosis disease duration, active inflammation, and demyelination. Journal: Stem Cells Translational Medicine.

3. Bijar, A., Khayati, R and Benavent, A.P. 2013 Increasing the contrast of the brain MR FLAIR images using fuzzy membership functions and structural similarity indices in order to segment MS lesions. https://doi.org/10.1371/journal.pone.0065469. g006.

4. Ji, Z., Sun, Q., Xia, Y., Chen, Q., Xia, D. and Feng, D., 2012. Generalized rough fuzzy c-means algorithm for brain MR image segmentation. Computer Methods and Programs in Biomedicine, 108, pp.644-655.

5. Henry, F. and McFarland, 2009. Examination of the role of mgnetic resonance imaging in multiple sclerosis: A problem-orientated approach. Annals of Indian Academy of Neurology.

6. Zhang, Y.D., Zhang, Y., Phillips, P. and Dong, Z., 2017. Synthetic minority oversampling technique and fractal dimension for identifying multiple sclerosis. Fractals, 25(4), p.1740010.

7. Sormani, M.P. and Bruzzi, P., 2013. MRI lesions as a surrogate for relapses in multiple sclerosis. A meta-analysis of randomised trials. Lancet Neurol., 12, pp.669-676.

8. Barkhof, F., 2002. The clinico-radiological paradox in multiple sclerosis revisited. Current Opinion in Neurology, 15, pp.239-245.

9. Lorenzo, D.G., Francis, S., Narayanan, S., Douglas, L., Arnold, D. and Collins, L., 2013. Review of automatic segmentation methods of multiple sclerosis white matter lesions on conventional magnetic resonance imaging. Medical Image Analysis, 17(1). 
10. Jason, E.H., Brian, N. and Sunanda, M., 2014. An Information Theoretic Approach via IJM to Segmenting MR Images with MS Lesions. IEEE 27th International Symposium on Computer-Based Medical Systems.

11. Van Leemput, K., Maes, F., Vandermeulen, D., Colchester, D. and Suetens, P., 2001. Automated segmentation of multiple sclerosis lesions by model outlier detection. IEEE TMI, 20(8), pp.677-689.

12. Anbeek, P., Vinchen, K.L., van Osch, M.J.P., Bisschops, M.J.P., and van der Grond, J., 2004. Probabilistic segmentation of white matter lesions in MR imaging. Neurolmage, 21, pp.1037-1044.

13. Souplet, J.C., Lebrun, C., Anyche, N. and Malandain, G., 2008. An automatic segmentation of T2-FLAIR multiple sclerosis lesions. 3D Segmentation in the Clinic: A Grand Challenge II: MS Lesion Segmentation.

14. Ait-Ali, L.S., Prima, S., Hellier, P., Carsin, B., Edan, G. and Barillot, C., 2005. STREM: A robust multidimensional parametric method to segment MS lesions in MRI. MICCAI 2005, LNCS, 3749, pp.409-416.

15. Garcia-Lorenzo, D., Prima, S., Morrissey, S.P. and Barillot, C., 2008. A robust expectation-maximization algorithm for multiple sclerosis lesion segmentation. Segmentation in the Clinic: A Grand Challenge II: Lesion Segmentation.

16. Bricq, S., Collet, Ch. and Armspach, J.P., 2008. Markovian Segmentation of 3D Brain MRI to Detect Multiple Sclerosis Lesions. IEEE international Conference on Image Processing, ICIP 2008, pp.733-736.

17. Neykov, N. and Neytchev, P., 1990. A robust alternative of the of the maximum likelihood estimator. COMPSTAT-Short Communications. Dubrovnik, Yugoslavia, pp.99-100.

18. Freifeld, O., Greenspan, H. and Goldberger, J., 2009. Multiple sclerosis lesion detection using constrained GMM and curve evolution. Int. J. of Biomedical Imaging, pp.1-13.

19. Warfield, S., 1996. Fast k-NN classification for multichannel image data pattern recognition. Pattern Recognit Lett., 17(7), pp.713-721.

20. Liu, J., Smith, C.D. and Chebrolu, H., 2009. Automatic Multiple Sclerosis Detection Based on Integrated Square Estimation. IEEE Computer Society Conference on Computer Vision and Pattern Recognition Workshops, Miami, FL. pp.31-38.

21. Prastawa, M. and Guido, G., 2008. Automatic MS lesion segmentation by outlier detection and information theoretic region partitioning. 3D Segmentation in the Clinic: A Grand Challenge II: MS Lesion Segmentation.

22. Wu, Y., Warfield, S.K., Tan, I.L., WessI III, W.M., Meier, D.S., Van Schijndel, R.A., Barkhof, F. and Guttmann, C., 2006. Automated segmentation of multiple sclerosis lesion subtype with multichannel MRI. Neurolmage, 32, pp.1025-1215.

23. Tu, Z., Narr, K., Dinov, I., Dollar, P., Thompson, P. and Toga, A., 2008. Brain anatomical structure parsing by hybrid discriminative/generative models. IEEE $T M I$.

24. Morra, J., Tu, Z., Toga, A. and Thompson, P., 2008. Automatic segmentation of MS lesions using a contextual model for the MICCAI grand challenge. $3 D$ Segmentation in the Clinic: A Grand Challenge II: MS Lesion Segmentation.

25. Anbeek, P., Vinchen, K.L. and Viergever, M.A., 2008. Antomated MS-lesion segmentation by K-nearest neighbor classification. 3D Segmentation in the Clinic: A Grand Challenge II: MS Lesion Segmentation.

26. Zhang, Y., Lu, S., Zhou, X., Yang, M., Wu, L., Liu, B., Phillips, P. and Wang, S., 2016. Comparison of machine learning methods for stationary wavelet entropy-based multiple sclerosis detection: Decision tree, k-nearest neighbors, and support vector machine. Simulation: Transactions of the Society for Modeling and Simulation International. Vol. 92(9), pp.861-871.

27. Benaichouche, A.N., Oulhadj, H. and Siarry, P., 2013. Improved spatial fuzzy c-means clustering for image segmentation using PSO initialization, Mahalanobis distance and post-segmentation correction. Digit. Signal Proc., 23, pp.1390-1400.

28. Bezdek, J.C., Hall, I. and Clarke, L., 1993. Review of MR image segmentation techniques using pattern recognition. Med. Phys., 20, pp.1033-1048.

29. Nikhil, R., Pal, Kuhu Pal, Keller, J.M. and Bezdek, J.C., 2005. A possibilistic fuzzy c-means clustering algorithm. IEEE Transactions on Fuzzy Systems, 13(4).

30. Saatchi, S. and Hung, C., 2007. Swarm intelligence and image segmentation, swarm intelligence, focus on ant and particle swarm optimization, 1 -Tech Education and Publishing.

31. Bezdek, J.C., 1973. Fuzzy mathematics in pattern classification. Ph.D Dissertation, Cornell University, Ithaca, NY.

32. Pang, W., Wang, K.P., Zhou, C.G. and Dong, L.J., 2004. Fuzzy Discrete Particleswarm Optimization for Solving Traveling Salesman Problem. In the Fourth International Conference on Computer and Information Technology, pp.796-800.

33. Pal, N.R., Pal, K. and Bezdek, J.C., 1997. A Mixed C-Means Clustering Model. Proceedings of the Sixth IEEE International Conference onFuzzy Systems, Vol. 1, pp.11-21.

34. Barkhof, F., 1999. MRI in multiple sclerosis: Correlation with expanded disability status scale (EDSS). Multiple Sclerosis, 5, pp.283-286.

35. El Dor, A., Lepagnot, J., Nakib, A. and Siarry, P., 2014. PSO-2S optimization algorithm for brain MRI segmentation. In Genetic and Evolutionary Computing. Springer, pp.13-22.

36. Selvi, V., Dr. Umarani, R., 2010. Comparative analysis of ant colony and particle swarm optimization techniques. Int. J. Comput. Appl. (0975-8887) 5(4).

37. Shi, Y. and Eberhart, R.C., 1999. Empirical Study of Particle Swarm Optimization. Vol. 3, pp.1945-1950.

38. Kennedy, J. and Eberhart, R., 1995. Particle swarm optimization. Proceedings IEEE International Conference on Neural Networks, Perth, Australia, Vol. 4, pp.1942-1948.

39. Ait-Ali, L.S., Prima, S., Edan, G. and Barillot, C., 2006. Longitudinal segmentation of MS lesions in multimodal brain MRI. 15eme Congres Francophone AFRIFIAFIA de Reconnaissance des Formes et Intelligence Artificielle (RFIA), Tours, France, Janvier.

40. Izakian, H. and Abraham, A., 2011. Fuzzy c-means and fuzzy swarm for fuzzy clustering problem. Expert Syst. Appl., 38(3), pp.1835-1838.

41. Surya Prabha, D. and Satheesh Kumar, J., 2016. Performance evaluation of image segmentation using objective methods. Indian Journal of Science and Technology, 9(8), DOI: 10.17485/ijst/2016/v9i8/87907.

42. Rehab, F. and Kader, A., 2011. Fuzzy particle swarm optimization with simulated annealing and neighborhood information communication for solving TSP, (IJACSA) International Journal of Advanced Computer Science and Applications, 2(5).

43. Wang, S.H., Cheng, H., Phillips, P. and Zhang, Y.D., 2018. Multiple sclerosis identification based on fractional fourier entropy and a modified jaya algorithm. Entropy, 20, p.254. 